\title{
LOCOMOTIVE NOISE REDUCTION
}

\author{
Nikita Nesterov $^{{ }^{*}}$ and Aleksei Bykov ${ }^{2}$ \\ ${ }^{1}$ Russian Research Institute of J SC Russian Railways, M oscow, Russian Federation \\ ${ }^{2}$ B auman M oscow State Technical U niversity, Power Engineering D epartment, 105005, M oscow, \\ Russian Federation
}

\begin{abstract}
This paper presents the results of the investigation of the noise characteristics of the dual-voltage mainline electric locomotive. The study identified noise levels generated by the locomotive equipment that violate regulations. The analysis of points, where the noise is above regulations, indicated the primary source of the elevated noise is the operation of the traction transformer. We proposed installing a noise barrier below the locomotive body to reduce noise and bring the locomotive in compliance with the noise regulations. This solution allowed reducing the locomotive noise and ensure its compliance with regulations.
\end{abstract}

\section{Introduction}

On Russian railways, most of the cargo and passengers are carried by electric locomotives. Compared to diesel-electric and other autonomous locomotives, electric locomotives produce less noise. However, studies show that these noise levels sometimes do not comply with regulations. Workplace noise disrupts communication and can be a factor contributing to a heightened risk of traumas and illnesses. Noise from the traction motor and the air compressor may affect the train driver's concentration and decision. Although the causal link between noise and hazards has not yet been established, noise is considered as a potential factor of risk of industrial traumas or, at least, the factor contributing to human errors. The reasons for this are as follows:

1) Noise drowns out alarm signals;

2) Noise disrupts communication and masks hazard alarms;

3) Noise distracts the drivers and reduces his/her concentration.

Regular high noise during work contributes to occupational illnesses among train crews. Sensorineural hearing loss is the number one occupational illness [1]. In conditions of industrial noise and vibration, all train drivers get medium or severe sensorineural hearing loss after 18 years of work [2]. Thus, research aiming to study noise characteristics and improve the noise protection of electric locomotives is relevant.

* Corresponding author: nesterov.nikita@vniizht.ru 


\section{Electric locomotive noise characteristics}

At the test loop of the SC "Scientific Research Institute of Railway Transport," external noise, noise in the driver cab and engine room of a mainline dual-voltage electric locomotive was measured. Studies of the characteristics of the electric locomotive external noise were presented in [3]. The studies showed that external noise requirements are not met when the locomotive is in stationary mode. Fig. 1 shows the distribution of the external noise levels of the stationed electric locomotive.

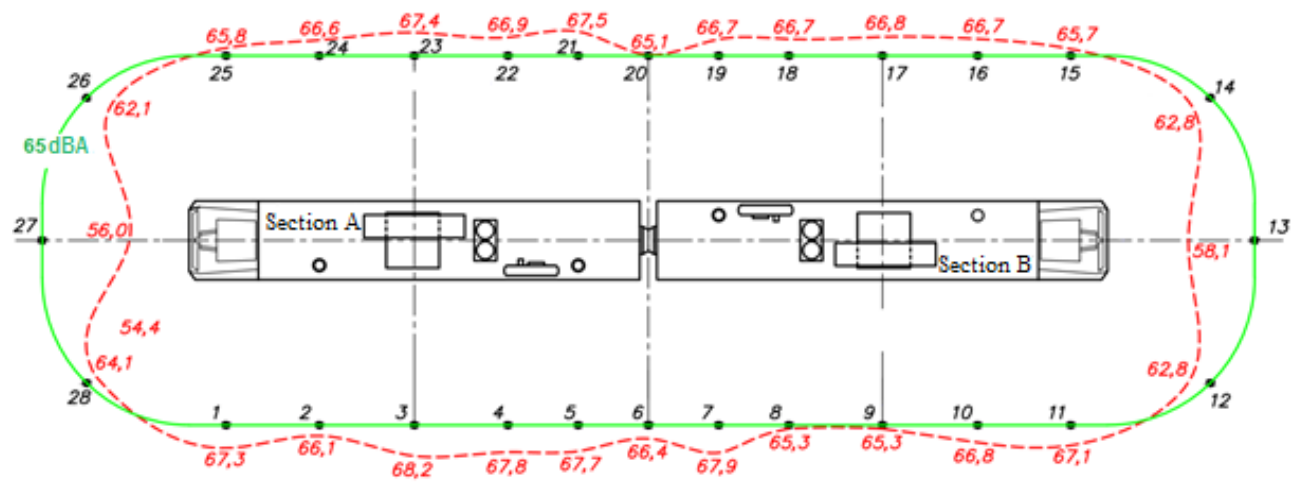

Fig. 1. Distribution of the external noise of the locomotive working in the AC mode.

The analysis of the noise level distribution revealed that the noise field generated by locomotive equipment is non-uniform. The highest noise is generated in points $3,17.23$, which are located opposite the traction transformer.

Also, sound pressure levels (SPL) were measured, Fig. 2, in the octave band in the points opposite the traction transformer $1 \mathrm{~m}$ away from it.

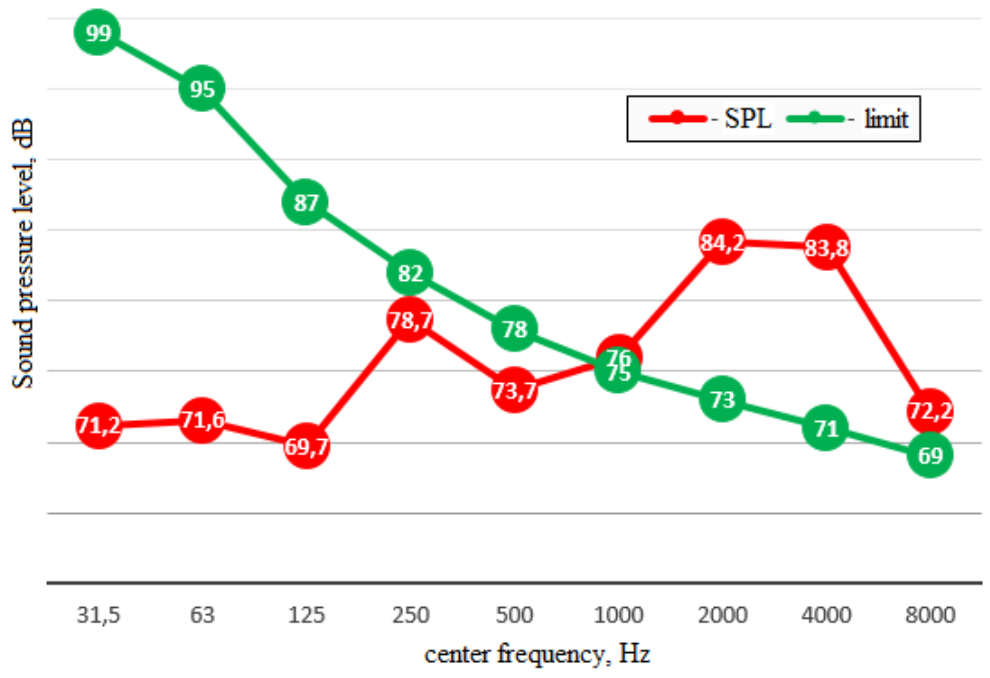

Fig. 2. Sound pressure levels measured in the octave bands opposite the traction transformer of the electric locomotive.

From $1000 \mathrm{~Hz}$ to $8000 \mathrm{~Hz}$, SPL is above the maximum permissible level by 1 to $12.8 \mathrm{~dB}$, accordingly. The analysis of the external noise levels in the measurement points where the 
noise levels violated the requirements indicated that the traction transformer of both sections is the main source of elevated external noise levels.

\section{Measures of reducing the noise of the electric locomotive}

During the traction transformer operation, its windings produce heat, so its active part is placed in a tank with transformer oil. The oil circulation removes heat. Oil electric pumps move the heated oil to the heat exchanged where the excess heat is given to the air stream created by the axial fan. The traction transformer noise is mainly related to magnetic noise and does not greatly depend on the cooling system's operation. The cause of magnetic noise is the magnetostriction effect occurring in the transformer core and causing bending oscillations of the core rods [4]. The core vibrations are transferred to the tank walls via oil and fixtures. After that, it is emitted as structural-borne noise transferred to the elements through the air. As reducing the noise by making structural changes of the transformer is not feasible, we proposed [5] installing a noise barrier (NB) below the body of the traction transformer, Fig. 3. This barrier is a partial noise barrier. Installing a full enclosure (cover) for the transformer part located under the body is not possible, because the transformer has to be accessible for maintenance. Besides, it must be able to remove heat in a natural way.

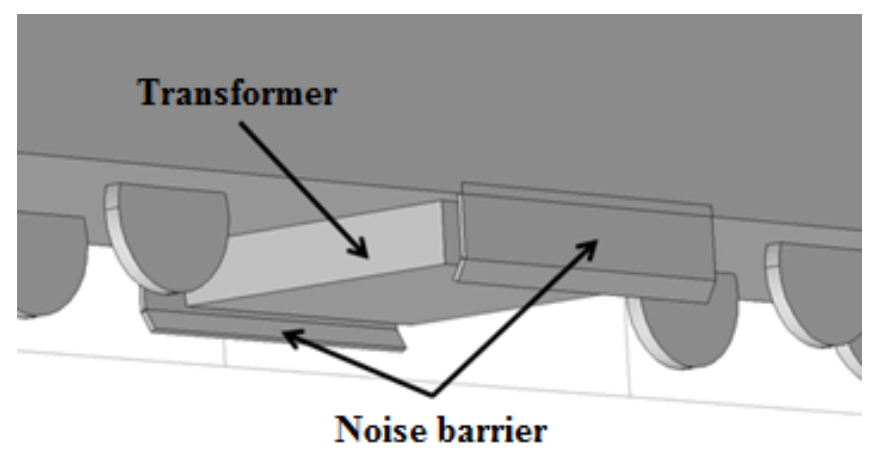

Fig. 3. Noise barrier location.

The noise barrier, Fig. 4, is a sandwich structure comprised of a steel sheet 1, shockabsorbing paste 2, sound-proofing material (SPM) 3, and a perforated steel sheet 4 .

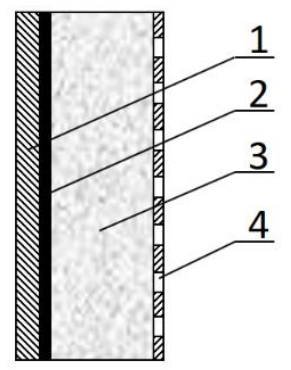

Fig. 4. Traction transformer noise barrier elements.

The main parameters of the proposed structure must be chosen based on the required noise-canceling efficiency. Parameters of the noise barrier include the distance from the transformer tank wall to the NB, length, height, and thickness of the steel sheet and the SPM sheet, as well as the acoustic reduction factor of the SPM. 


\section{Calculation of the sound barrier parameters}

To reduce the traction transformer noise, the noise barrier has to be selected. Its efficiency $\Delta L_{\mathrm{NB}}$ must satisfy the following condition:

$$
\Delta L_{\mathrm{NB}} \geq L_{c p}-L_{p e r m}, \mathrm{~dB}
$$

where $L_{c p}$ is the sound pressure level in the octave band in the calculation point (CP) measured opposite the electric locomotive traction point, Fig. $2 ; L_{\text {perm }}$ is the maximum permissible level of the sound pressure on the workplace, according to [6].

So, the required NB efficiency will correspond to the values presented in Table 1.

Table 1. Results of calculating required NB efficiency.

\begin{tabular}{|c|c|c|c|c|c|c|c|c|c|}
\hline \multirow{2}{*}{ Parameter } & \multicolumn{7}{|c|}{ Sound pressure levels, dB, with octave band center frequency, Hz } \\
\cline { 2 - 10 } & $\mathbf{3 1 . 5}$ & $\mathbf{6 3}$ & $\mathbf{1 2 5}$ & $\mathbf{2 5 0}$ & $\mathbf{5 0 0}$ & $\mathbf{1 0 0 0}$ & $\mathbf{2 0 0 0}$ & $\mathbf{4 0 0 0}$ & $\mathbf{8 0 0 0}$ \\
\hline$L_{\mathrm{cp}}$ & 71.2 & 71.6 & 69.7 & 78.7 & 73.7 & 76 & 84.2 & 83.8 & 72.2 \\
\hline$L_{p e r m}$ & 99 & 95 & 87 & 82 & 78 & 75 & 73 & 71 & 69 \\
\hline$\Delta L_{\mathrm{NB}}$ & - & - & - & - & - & 1 & 11.2 & 12.8 & 3.2 \\
\hline
\end{tabular}

Further calculations determined the NB parameters described above. To determine the required parameters, the noise barrier was modeled using finite element modeling in COMSOL Multiphysics. Such an approach in recent times is increasingly used [7-11]. This modeling method allows presenting a discrete space as a finite set of elementary volumes. For each finite element, an approximate equation relative to the unknown amplitude is constructed. By combining the solutions for all finite elements, sound pressure levels in the elementary volume are acquired.

The classical three-dimensional representation of the noise barrier can be simplified, i.e., barrier rounding by acoustic waves can be neglected. Thus, a two-dimensional model is acquired where the barrier is assumed to have infinite length, and the sound can only penetrate it in one direction. This approach can significantly reduce the number of finite elements in the model because meshing a two-dimensional model is much simpler compared to a three-dimensional model. The number of elements was around 20 million to ensure adequate calculation accuracy in a wide frequency band.

The model of the noise barrier is a parallelepiped whose length is $1 \mathrm{~m}$ greater than the transformer length, and the length was varied to find the optimal variant of the noise barrier efficiency. For metallic surfaces of the noise barrier, an absolute rigid wall boundary condition was applied. All locomotive elements represented in the model were treated as absolutely rigid. The transformer noise was modeled as radiation from planer sources, i.e., the surfaces forming the transformer model. The radiation amplitude was $1 \mathrm{~Pa}$.

The ground surface has some absorbing properties corresponding to the absorption of the sound by a layer of crushed stone. The sound absorption coefficient values $\alpha$, Table 2 , were selected according to [12].

Table 1. Sound absorption coefficients.

\begin{tabular}{|c|c|c|c|c|c|c|c|c|}
\hline \multirow{2}{*}{\begin{tabular}{c} 
Ground surface type \\
\cline { 2 - 9 }
\end{tabular}} & 63 & 125 & 250 & 500 & 1000 & 2000 & 4000 & 8000 \\
\hline Crushed stone & 0.1 & 0.1 & 0.2 & 0.2 & 0.3 & 0.3 & 0.3 & 0.2 \\
\hline
\end{tabular}


First, a model without the NB was simulated. The simulation yielded values of SPL in calculation points, Fig. 5, and diagrams of distribution of the sound pressure in the considered simulation domain. For demonstration purposes, Fig. 6 shows the initial distribution of the sound pressure levels around the locomotive in the transverse plane for the frequency of 4000 $\mathrm{Hz}$.

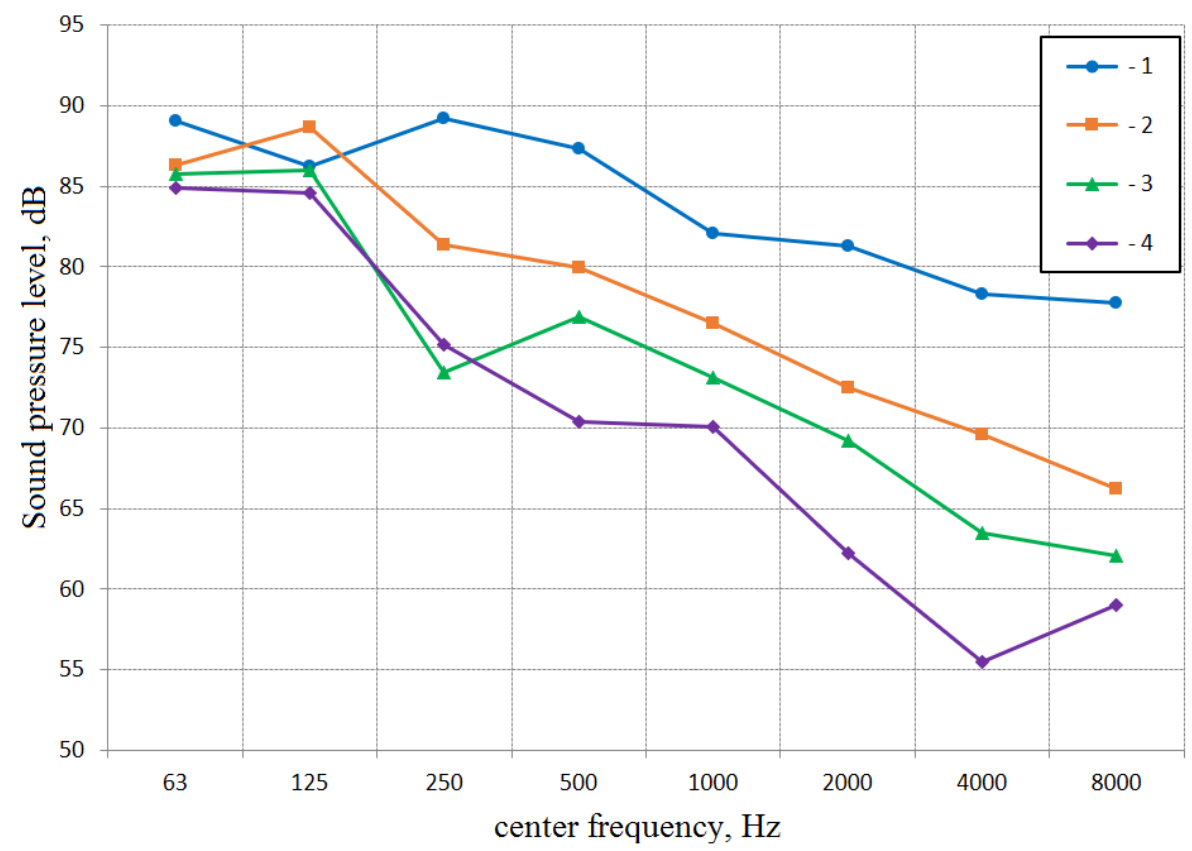

Fig. 5. Simulated SPL in the calculation points: 1 - model without NB; 2 - model with NB without SPM; 3 - model with NB and SPM; 4 - model with NB and SPM (height $+150 \mathrm{~mm}$ ).

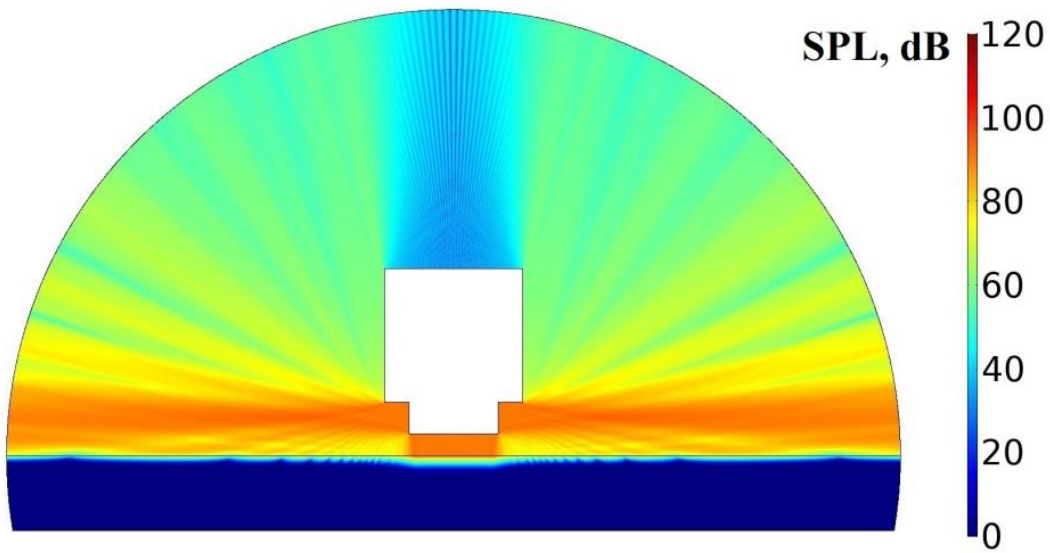

Fig. 6. Original distribution of the sound pressure levels for the frequency of $4000 \mathrm{~Hz}$ for the crushed stone ground surface.

The next stage of the simulation was the determination of the sound pressure levels in the calculation points for the model with the NB. The first model of NB was an absolute rigid screen with a thickness of $3 \mathrm{~mm}$ aligned with the sidewall of the locomotive body, Fig. 3 . The NB height was set equal to that of the traction transformer. The second model of the NB 
was a sandwich structure with a rigid screen and the $100 \mathrm{~mm}$ SPM layer on the interior side. For the third model, the NB height was increased by $150 \mathrm{~mm}$. Simulated SPL values in the calculation points for three NB models are shown in Fig. 5. The simulation showed that the NB model in the form of a rigid screen does not have the required efficiency. For the second model, there was increased transformer sound reduction efficiency of 12 and $15 \mathrm{~dB}$ at 2000 and $4000 \mathrm{~Hz}$, accordingly. This barrier configuration has the required efficiency; however, it should be designed with a safety margin given that transformer noise may be higher than measured at some operating conditions. So, to reduce the noise of the traction transformer, the sandwich structure NB with the SPM and the height $150 \mathrm{~mm}$ greater than the transformer height was selected. Fig. 7 shows the color sound distribution plot for the model with the NB at the frequency of $4000 \mathrm{~Hz}$.

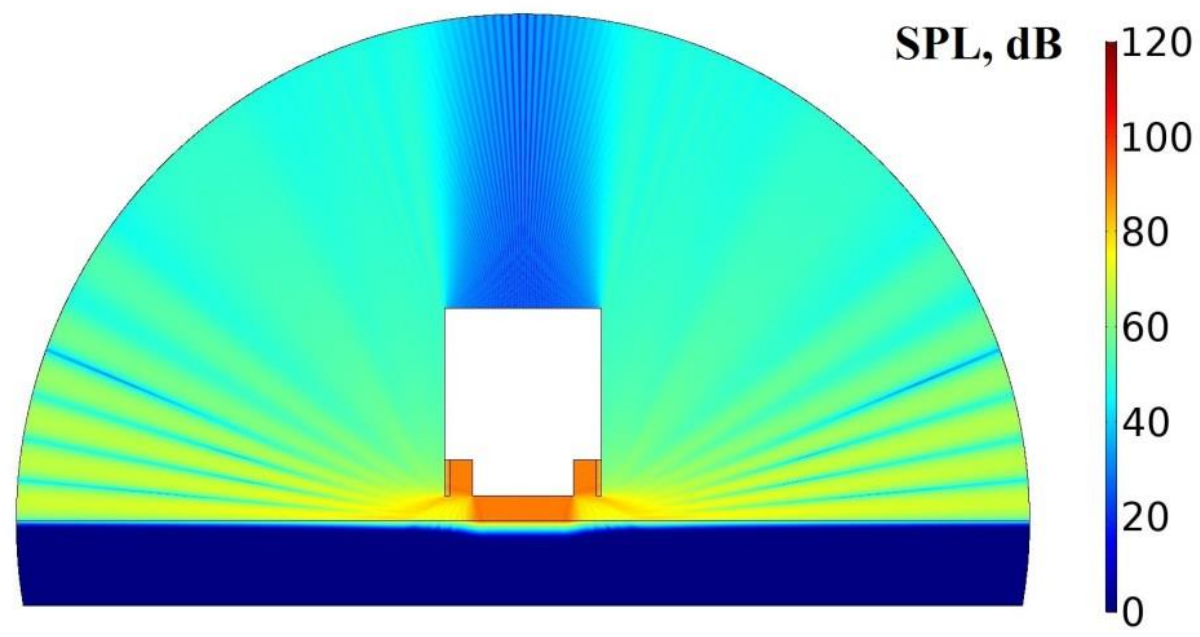

Fig. 7. SPL distribution for the model with the NB at $4000 \mathrm{~Hz}$; the ground surface is crushed stone.

Also, we simulated the case of the absolutely rigid ground surface (such a surface has a zero-reflection coefficient). This case is possible when the locomotive is in a train yard. For example, Figure 8 shows the $4000 \mathrm{~Hz}$ SPL distribution for the NB model when the ground surface is absolutely reflecting.

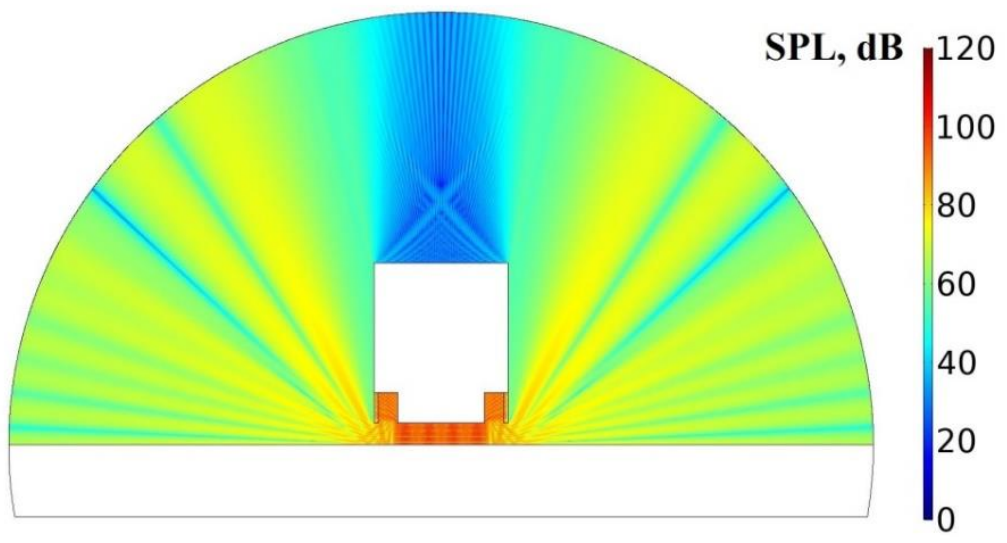

Fig. 8. SPL distribution at the frequency of $4000 \mathrm{~Hz}$ for the absolute rigid ground surface. 
Based on the simulation results for different ground surface types, the efficiency of the NB for the rigid surface is lower. This fact confirms that the sound efficiency of the NB must have higher than the required calculated efficiency values.

After the NB was installed at the locomotive, the external noise levels were measured again [3]. Fig. 9 shows the measurement results.

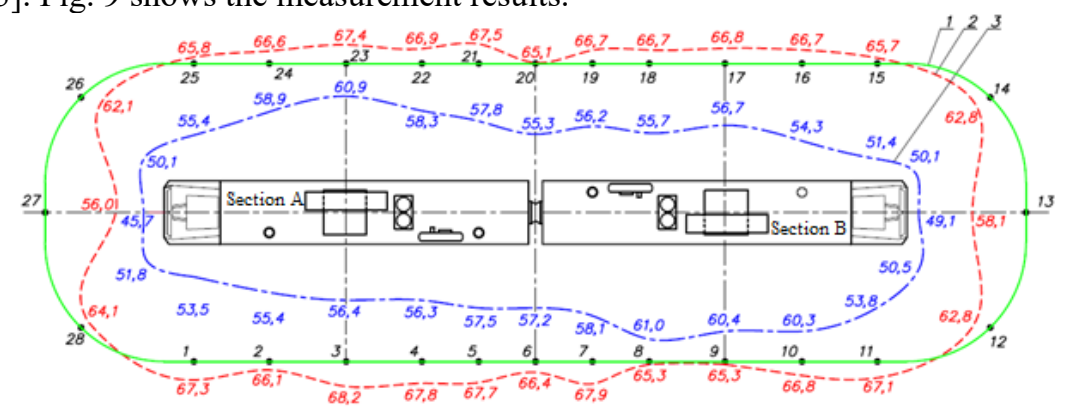

Fig. 9. External noise distribution of the stationary electric locomotive working in the AC mode: 1 maximum permissible external noise level $\mathrm{LA}=65 \mathrm{dBA}$ of a stationary electric locomotive; 2 locomotive noise level distribution without the noise barrier; 3 - locomotive noise level distribution with the noise barrier.

As a result of installing the noise barrier, the noise of the locomotive traction noise in the measurement points was reduced by $3.5-13.5 \mathrm{dBA}$. This measure allowed bringing the locomotive in compliance with requirements.

\section{Conclusion}

As a result of the investigations to reduce electric locomotive traction transformer noise levels, we proposed installing a noise barrier with a required soundproofing efficiency under the locomotive body. The parameters and configuration of the noise barrier were selected based on simulation in COMSOL Multiphysics. Installing the noise barrier reduced the external noise and brought the locomotive in compliance with the noise regulations.

\section{References}

1. T. D. Karetskaya, V. F. Pfaf, Occupational illnesses at the railway transport, Russian J ournal of Occupational $\mathrm{H}$ eal th and Industrial Ecology, 1, 1 - 5 (2015)

2. A. E. Rybnikova, R. A ., Berg Influence of industrial noise and vibration on development of occupational sensorineural hearing loss of train drivers, Siberian J ournal of Life Sciences and A griculture, 10, 104 - 106 (2010)

3. M. Y u. Noskov, N. S. N esterov, Y u. A. K hlobystov, Investigation of characteristics of the external noise of the dual-voltage electric locomotive, V estnik of the Railway Research Institute, 78(2), 105 - 113 (2019)

4. Engineering acoustics handbook, eds. M. K hekl and K h.A. M uller. Leningrad.: Sudostroenie, (1980)

5. Nesterov N.S. Investigation of the external noise of the new generation electric locomotive, in proceedings of the third A II-Russian conference of young scientists and specialists “Acoustics of the Environment”(A SO-2018), 18 M ay 2018, M OSCow, Russia (2018) 
6. M ethod of conducting special assessment of working conditions approved by the order of the M inistry of Labor and Social Protection of the Russian Federation dated 24 January 2014. No 33n (2014)

7. A. I.Komkin, M. A . M ironov, S. I. Y udin, Eigenfrequency of a Helmholtz Resonator at the Wall of a Rectangular Duct, A coustical Physics, 60(2),142-147 (2014)

8. A . I. Komkin, A . I. Bykov, Inertial attached neck length of Helmholtz resonators, A coustical Physics, 62(3),269-279 (2016)

9. M. Kalugin et al., Using big analytics tools in performance of gas dynamics and acoustics tasks, Herald of the B auman M oscow State Technical U niversity. Series Natural Sciences, 3, 32-47 (2018)

10. A . E pikhin, M. K raposhin, K. V atutin, The numerical simulation of compressible jet at low Reynolds number using OpenFOAM, E3S W eb of Conferences. EDP Sciences, 128, 10008 (2019)

11. S. M. Sivachev, L. L. M yagkov, Thermomechanical Fatigue Analysis of Diesel Engine Piston: Finite Element Simulation and Lifetime Prediction Technique, International Conference on Industrial Engineering, Springer, Cham, Switzerland, (2019)

12. D. A. Kuklin, The issue of reducing external noise of trains in the source and on the propagation path: doctoral dissertation: 01.04.06. B altic State Technical U niversity, Saint-Petersburg (2016) 